\title{
Sini-san improves duodenal tight junction integrity in a rat model of functional dyspepsia
}

\author{
Xiongfei Chang ${ }^{1,2}$, Luqing Zhao ${ }^{2}$, Jiajia Wang ${ }^{2}$, Xiaofang Lu $^{2}$ and Shengsheng Zhang ${ }^{2^{*}}$ (D)
}

\begin{abstract}
Background: Recent reports have demonstrated that impaired barrier function and local microinflammation in the duodenal mucosa contribute to the pathogeneses of functional dyspepsia (FD). Thus, restoring normal barrier integrity becomes a potential therapeutic strategy in the treatment of FD. Sini-San (SNS) is a traditional Chinese prescription that exhibits therapeutic effects in FD, but the underlying mechanisms remain not well understood.

Methods: FD rats were established by tail clamping method and the therapeutic effect of SNS was evaluated by measuring the visceral sensitivity and gastric compliance. Transepithelial electrical resistance (TEER) that reveals epithelial barrier integrity was measured by Ussing chamber. The expression of tight junction (TJ) proteins, occludin and claudin-1, in the duodenum was determined by Western blot and immunofluorescence. The amount of tumor necrosis factor alpha (TNF-a) and interferon gamma (INF- $\gamma$ ) in duodenal mucosa was detected by enzyme-linked immune sorbent assay (ELISA). The mRNA level of transient receptor potential vanilloid type 1 (TRPV1) was measured by quantitative real time-polymerase chain reaction (qPCR).
\end{abstract}

Results: SNS could improve gastric compliance and attenuate visceral hypersensitivity (VH) in FD rats. TEER was decreased in FD rats, but treatment with SNS restored normal level of TEER and the expression of occludin and claudin-1 in FD rats. In addition, SNS administration ameliorated FD-associated increase in the production of TNF- $a$, IFN- $\gamma$ and the expression of TRPV1.

Conclusions: The therapeutic effect of SNS on FD is at least partially through improvement of TJ integrity and attenuation of FD-associated low-grade inflammation in the duodenum. Our findings highlight the molecular basis of SNS-based treatment of FD in human patients.

Keywords: Sini-san, Functional dyspepsia, Duodenum, Tight junction, Pro-inflammatory cytokine

\section{Background}

Functional dyspepsia (FD) is a common functional gastrointestinal disorder affecting $10-30 \%$ of the population globally [1]. FD is characterized by upper abdominal pain, early satiety and belching. The underlying pathogenesis is complicated and largely unknown. Potential causes are visceral hypersensitivity $(\mathrm{VH})$, decreased gastric compliance and abnormal gastric motility. It was proposed there are no structural changes associated with

\footnotetext{
* Correspondence: zss2000@sohu.com

Xiongfei Chang and Luqing Zhao share first authorship.

Xiongfei Chang and Luqing Zhao contributed equally to this study.

${ }^{2}$ Digestive Disease Center, Beijing Hospital of Traditional Chinese Medicine

Affiliated to Capital Medical University, Beijing, China

Full list of author information is available at the end of the article
}

FD in the upper gut, but recent studies have shown that duodenal barrier function is impaired and low-grade inflammation is present in the duodenum of FD patients. These findings recognize that the duodenum has a key role in the pathogenesis of FD $[2,3]$, and propose that duodenal barrier dysfunction and local low-grade inflammation are potential pathogenic factors for FD.

The intestinal barrier plays a critical role in preventing the translocation of noxious substances from the gut lumen to the submucosa, and the tight junction (TJ) proteins are key structural factors of this barrier. Dysfunction in intestinal barrier causes abnormal penetration of toxic substances, which results in increased local expression of inflammatory factors or infiltration of 
inflammatory cells. Many studies have proved that this pathological process often occurs in inflammatory bowel disease [4].

Sini-San (SNS) is a traditional Chinese prescription that has been widely applied in the treatment of various gastrointestinal diseases, including FD [5-7]. SNS is composed of four herbs, Chaihu (Radix Bupleuri Chinensis), Baishao (Radix Paeoniae Alba), Zhishi (Fructus Aurantii Immaturus), and Gancao (Radix Glycyrrhizae). It was shown that SNS could reduce the permeability of intestinal mucosa [8], and pharmacological studies also demonstrated that some extracts of these herbs, such as $18 \beta$-glycyrrhetinic acid [9], could also protect or repair intestinal epithelial TJ. However, it is unknown whether SNS plays a therapeutic role in FD by repairing tight junction integrity in the duodenum.

The aims of this study were to evaluate the therapeutic effect of SNS in FD and to determine whether SNS regulates the expression of $\mathrm{TJ}$ proteins thereby improving duodenal barrier function.

\section{Methods \\ SNS preparation}

SNS consists of Chaihu (Radix Bupleuri Chinensis, derived from Bupleurum chinense DC, voucher number 16012002), Baishao (Radix Paeoniae Alba, derived from Paeonia lactiflora Pall, voucher number 16040201), Zhishi (Fructus Aurantii Immaturus, derived from Citrus aurantium L, voucher number 16011370), and Gancao (Radix Glycyrrhizae, derived from Glycyrrhiza uralensis Fisch, voucher number 15091001) with a ratio of 1:1:1:1.

These raw herbs were purchased from Beijing Xinglin Pharmaceutical Company and were identified as eligible medicinal material. SNS was prepared by the Beijing Hospital of Traditional Chinese Medicine Affiliated to Capital Medical University. Specifically, a total weight of $400 \mathrm{~g}$ of the above raw herbs was mixed and impregnated in $2400 \mathrm{ml}$ distilled water for $30 \mathrm{~min}$. Then the medical materials were boiled for $30 \mathrm{~min}$ and $400 \mathrm{ml}$ SNS preparation was harvested. This procedure was repeated, and a final volume of $800 \mathrm{ml}$ was obtained from each $400 \mathrm{~g}$ herbs. The decoction was stored at $4{ }^{\circ} \mathrm{C}$ until the experiment. The major components of SNS decoction were saikosaponins, peoniflorin, naringin and glycyrrhizic acid [10-12].

\section{Animals}

Thirty six healthy male Sprague-Dawley rats (SPF grade) weighing $200 \pm 20 \mathrm{~g}$ were bought from Beijing Vital River Laboratory Animal Technology Company. The rats were housed in cages maintained under a 12-h light/12-h dark cycle with the room temperature of $22 \pm 1{ }^{\circ} \mathrm{C}$ and a humidity of $65-70 \%$. All of the rats had free access to food and water. Twenty four rats were used to establish the FD model by tail clamping approach as previously described [13], and the remaining 12 rats were divided into control group. In brief, every four rats were kept in a cage, and a surgical forcep was used to clamp the distal one third of the tail. This tail clamping was practiced every $4 \mathrm{~h}$ with a duration of $30 \mathrm{~min}$, and 3 times per day for 7 days. The FD rats were then randomly divided into two groups that receive SNS or water as a vehicle control. Equal volume of SNS or water was given through gavage at the dose of $1 \mathrm{ml} /$ $100 \mathrm{~g}$ body weight for 7 consecutive days. At the end of treatment, 6 rats of each group were used to evaluate the gastric compliance and sensitivity. The remaining 6 rats in each group were anesthetized using $2 \%$ pentobarbital sodium and the duodenum was excised for the following experiments.

Animal experiments were performed in accordance with the Guide for the Care and Use of Laboratory Animals published by the National Institutes of Health (NIH Publications No. 85-23, revised 1996) and approved by the Animal Care and Use Committee of China Academy of Traditional Chinese Medicine.

\section{Gastric distension procedure and myoelectricity record}

Gastric compliance and sensitivity were evaluated by using barostat and electrophysiological recorder as reported previously $[14,15]$. Briefly, the rats were temporarily anesthetized by isoflurane. A polyethylene balloon (maximum volume $20 \mathrm{ml}$ ) with a polyvinyl tube was introduced from the mouth to the stomach, and two electrodes were fixed into the trapezius of the rat. The balloon and the electrodes were connected to the barostat and electrophysiological recorder respectively. The experiments began when the rats were conscious. The pressure of the balloon was increased stepwise, 20, 40, $60,80 \mathrm{mmHg}$, with duration of $30 \mathrm{~s}$ and at an interval of 3 min between distensions. The volume of the balloon was recorded by the Protocol PlusDeluxe 9.6 R (G\&J Electronics, Canada). Simultaneously, the myoelectricity was recorded by the LabChart (AD instrument, Australia). The gastric compliance was calculated based on the volume changes per mmHg during the distension, while the gastric sensitivity is presented as the rate of change root mean square of the myoelectricity.

\section{Ussing chamber experiment}

The duodenal epithelial barrier integrity was examined by Ussing chamber system (Physiologic Instruments, USA). After the muscular layer and serosa were removed, the duodenal mucosal layer was mounted in the Ussing chamber as described previously [2]. The chambers were filled with $5 \mathrm{ml}$ Krebs buffer that was maintained at $37{ }^{\circ} \mathrm{C}$ and perfused with $\mathrm{O}_{2} / \mathrm{CO}_{2}$ (95/5\%) continuously. After a 30 -min equilibration period, a 
constant electric pulse of $1 \mathrm{mV}$ every $60 \mathrm{~s}$ with duration of $0.2 \mathrm{~s}$ started and the voltage deflection was recorded every 15 min over 1 -h duration. The average voltage deflection of the four time points was calculated and Transepithelial electrical resistance (TEER) is presented as $\Omega / \mathrm{cm}^{2}$.

\section{Western blot}

The duodenum was homogenized by using a bead mill (Biospec, USA) in RIPA buffer containing $1 \mathrm{mM}$ PMSF (Solarbio, China). The BCA assay was used to measure the protein concentration. Equal amount of total proteins $(80 \mu \mathrm{g} /$ lane) was loaded on SDS-PAGE gel and transferred to PVDF membrane after separation. The membrane was then incubated with the following primary antibodies: anti-occludin (1:400, Santa Cruz Biotechnology), anti-claudin-1(1:2000, Abcam), and anti$\beta$-actin (1:1500, ZSGB-BIO) at $4{ }^{\circ} \mathrm{C}$ overnight. After three 10-min washing with PBS (supplemented with $0.1 \%$ Tween 20), the membrane was incubated with appropriate secondary antibodies: HRP-conjugated goat anti-mouse, rabbit anti-goat or goat anti-rabbit (both 1:5000, ZSGB-BIO) antibody. The protein bands on the membrane were visualized following incubation with enhanced chemiluminescence substrate and the protein levels were quantified with Image J software.

\section{Immunofluorescence}

Duodenum was embedded in OCT and frozen sections at $6-\mu \mathrm{m}$ thickness were processed with freezing microtome (ThermoFisher, USA). Sections were incubated with anti-occludin (1:50, Santa Cruz Biotechnology) or anti-claudin-1 (1:40, Abcam) at $4{ }^{\circ} \mathrm{C}$ overnight. Following three 10-min washing, the sections were then incubated with rabbit anti-goat or goat anti-rabbit (1:100, ZSGBBIO) secondary antibody at room temperature for $45 \mathrm{~min}$. The slides were mounted with mounting medium with DAPI (ZSGB-BIO) and signal was examined under fluorescence microscope (OLYMPUS DP71, Japanese).

\section{Enzyme-linked immunesorbent assay (ELISA)}

Samples were homogenized and the supernatants were collected. Total protein concentration was measured by $\mathrm{BCA}$ assay. The concentrations of tumor necrosis factor alpha (TNF- $\alpha$ ) and interferon gamma (IFN- $\gamma$ ) were detected with Rat TNF- $\alpha$ and IFN- $\gamma$ ELISA kits (Cusabio, China) respectively according to the manufacturer's instructions. Briefly, TNF- $\alpha$ or IFN- $\gamma$ antibody-coated plates were incubation with the above collected supernatants, followed by addition of biotin-conjugated antibody and avidin-conjugated HRP prior to incubation with TMB substrate. The signal was detected by a microplate reader (BioTek Instruments, USA) at $450 \mathrm{~nm}$, and the concentration of TNF- $\alpha$ and IFN- $\gamma$ is presented as $\mathrm{pg} / \mathrm{mg}$ protein.
Quantitative real-time polymerase chain reaction ( $q P C R$ ) The RNA was extracted from the duodenum using the TRIZOL reagent (Thermo Fisher Scientific, USA) and $5 \mu \mathrm{g}$ of total RNA were reverse transcribed to cDNA according to the manufacturer's instructions. Equal amount of cDNA was used to examine the expression of transient receptor potential vanilloid type 1 (TRPV1) ( $\beta$ actin as an internal control) by using the SYBR Master Mix (Promega, USA) and the CFX 96 Real-time PCR System (BIO-RAD, USA) under the conditions of initial activation at $95{ }^{\circ} \mathrm{C}$ for $2 \mathrm{~min}, 40$ cycles of denaturation at $95{ }^{\circ} \mathrm{C}$ for $15 \mathrm{~s}$, annealing/extension at $60{ }^{\circ} \mathrm{C}$ for $1 \mathrm{~min}$. The primer sequences are: $\beta$-actin (predicted size: 150 bp): sense 5' -AGTTGCGTTACACCCTTTC-3', antisense 5'-CACCTTCACCGTTCCAGT-3'; TRPV1 (predicted size: $262 \mathrm{bp}$ ): sense 5'-GACATGCCACCCAGCAGG-3', antisense 5' -TCAATTCCCACACAC CTCCC-3'.

\section{Statistical analysis}

All data were expressed as mean \pm SEM and one way analysis of variance (ANOVA) was used to test the statistical significance among the groups. Statistical significance was defined by $P<0.05$. SPSS 16.0 was used for all statistical analyses.

\section{Results}

SNS ameliorates visceral hypersensitivity (VH) and enhances gastric compliance in FD rats

$\mathrm{VH}$ is an underlying cause of FD symptoms, whereas decreased gastric compliance is known to be a consequence of FD. Herein, we found that FD rats elicited VH under all distention pressures of $20 \mathrm{mmHg}(29.22 \pm 3.59$ vs. $11.91 \pm 1.68, P<0.01), 40 \mathrm{mmHg}(77.31 \pm 9.30$ vs. $33.44 \pm 6.64, P<0.01), 60 \mathrm{mmHg}(213.39 \pm 30.65$ vs. $122.08 \pm 21.28, P<0.05)$ and $80 \mathrm{mmHg}(251.32 \pm 40.72$ vs. $126.40 \pm 24.61, P<0.05)$ compared with the control group (Fig. 1a). Importantly, SNS treatment significantly decreased visceral sensitivity in FD rats at $20 \mathrm{mmHg}$ $(16.81 \pm 3.05$ vs. $29.22 \pm 3.59, P<0.01), 40 \mathrm{mmHg}$ $(35.00 \pm 5.80$ vs. $77.31 \pm 9.30, P<0.01), 60 \mathrm{mmHg}$ $(106.54 \pm 11.51$ vs. $213.39 \pm 30.65, P<0.01)$, and $80 \mathrm{mmHg}(132.50 \pm 20.93$ vs. $251.32 \pm 40.72, P<0.05)$ (Fig. 1a). On the other hand, compared with the control animals FD rats showed a significant decrease in gastric compliance at $40 \mathrm{mmHg}(0.18 \pm 0.01$ vs. $0.29 \pm 0.01$, $P<0.01), 60 \mathrm{mmHg}(0.21 \pm 0.01$ vs. $0.31 \pm 0.01$, $P<0.01)$ and $80 \mathrm{mmHg}(0.22 \pm 0.01$ vs. $0.29 \pm 0.01$, $P<0.01)$, but not at $20 \mathrm{mmHg}(0.21 \pm 0.01$ vs. $0.25 \pm 0.01, P>0.05$ ) (Fig. 1b). Treating FD rats with SNS significantly improved gastric compliance under the pressures of $40 \mathrm{mmHg}(0.29 \pm 0.01$ vs. $0.18 \pm 0.01$, $P<0.01), 60 \mathrm{mmHg}(0.30 \pm 0.01$ vs. $0.21 \pm 0.01$, $P<0.01)$ and $80 \mathrm{mmHg}(0.29 \pm 0.01$ vs. $0.22 \pm 0.01$, 


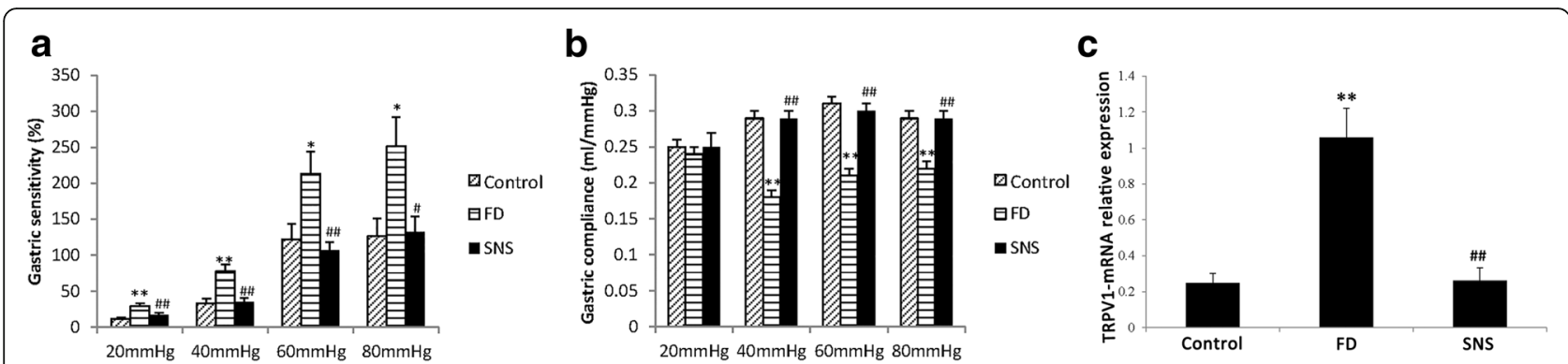

Fig. 1 Visceral sensitivity (a), gastric compliance (b) and mRNA expression of TRPV1 (c). The model group had elevated visceral sensitivity and decreased gastric compliance compared with the control group. SNS treatment was effective to recover the visceral sensitivity and gastric compliance. The mRNA level of TRPV1 was notably increased in the duodenum of the model group compared with the control group, and the SNS treatment decreased the expression of TRPV1. All data were represented as mean \pm SEM ( 6 rats in each group). ${ }^{*} P<0.05$ compared with the control group; ${ }^{* *} P<0.01$ compared with the control group; ${ }^{\#} P<0.05$ compared with the model group. ${ }^{\# \#} P<0.01$ compared with the model group

$P<0.01$ ) (Fig. 1b). These findings thus suggest that SNS improves FD symptoms, which is probably through attenuation of gastric hypersensitivity. To further confirm that SNS improves $\mathrm{VH}$, we analyzed the expression of TRPV1, which is a key factor that meditates VH. The qPCR result showed that the mRNA level of TRPV1 was significantly increased in the duodenum of FD rats as compared to the control group $(1.06 \pm 0.16$ vs. $0.25 \pm 0.05, P<0.01$ ) (Fig. 1c). Importantly, SNS treatment significantly reduced TRPV1 expression in FD rats $(0.26 \pm 0.07$ vs. $1.06 \pm 0.16, P<0.01)$.

\section{SNS improves TEER in FD rats}

Impaired duodenal function is known to cause gastric hypersensitivity and FD symptoms [16-18]. It has recently been shown that duodenal barrier function is compromised in FD. We asked whether TEER is altered in our FD rats, and assessed the potential effects of SNS on TEER regulation. As shown in Fig. 2, TEER values as detected by Ussing chamber was significantly decreased in the duodenum of FD rats as compared with the control group (18.71 \pm 1.51 vs. $40.06 \pm 0.77, P<0.01)$. SNS treatment remarkably enhanced the TEER level of FD rats $(37.70 \pm 1.84$ vs. $18.71 \pm 1.51, P<0.01)$, indicating that SNS can repair duodenal epithelial barrier function.

\section{SNS increases the expression of TJ proteins in FD rats}

$\mathrm{TJ}$ proteins create the first line of paracellular barrier, protecting the mucosa from the external environment. We next determined whether SNS restores TEER by regulating of the expression of $\mathrm{T}$ J proteins. Occludin and claudin-1 were examined in the present study because of their essential roles in maintaining a tight barrier [19]. Western blot analysis showed that the expression levels of occludin and claudin-1 were significant lower in the FD rats as compared with the control group (occludin: $0.031 \pm 0.008$ vs. $0.123 \pm 0.009, P<0.01$; claudin- 1 : $0.007 \pm 0.001$ vs. $0.062 \pm 0.001, P<0.01$ ) (Fig. 3).
Moreover, immunofluorescence staining showed a decrease in the expression of both occludin (Fig. 4a) and claudin-1 (Fig. 4b) in the FD group, which was restored in response to SNS treatment. These findings implicate that SNS enhances epithelial barrier function at least in part through the up-regulation of $\mathrm{TJ}$ proteins.

\section{SNS attenuates the expression of pro-inflammatory cytokines in FD rats}

Impaired duodenal barrier function exposes the body to luminal environment where commensal bacteria inhabit, which can cause inflammatory response in the mucosa. In fact, several studies have demonstrated the presence of low-grade inflammation in the duodenum of FD patients $[20,21]$. In this study, we assessed whether application of SNS in FD rats modulates the inflammatory state by examining the expression of typical proinflammatory cytokines, TNF- $\alpha$ and IFN- $\gamma$.

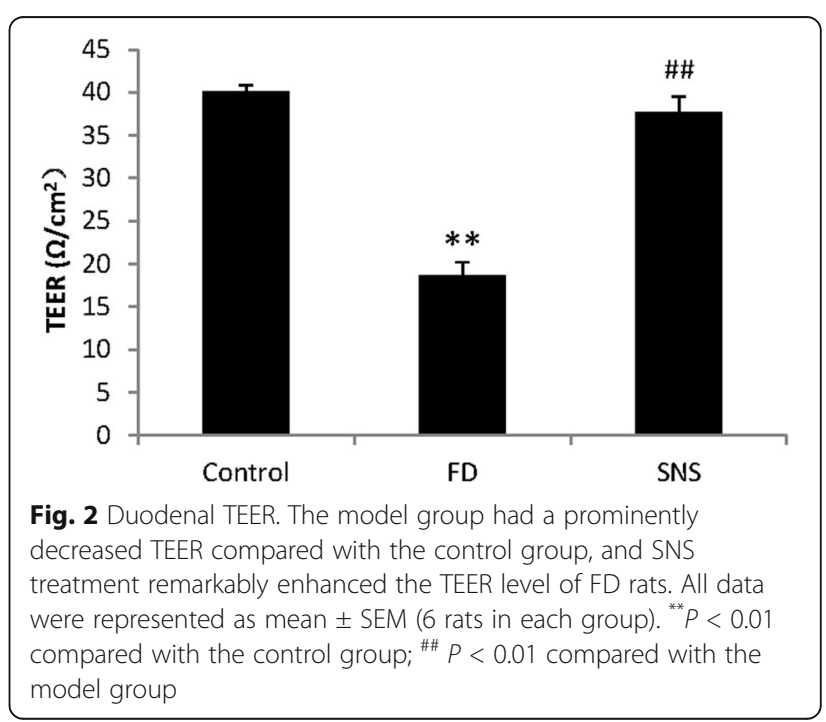




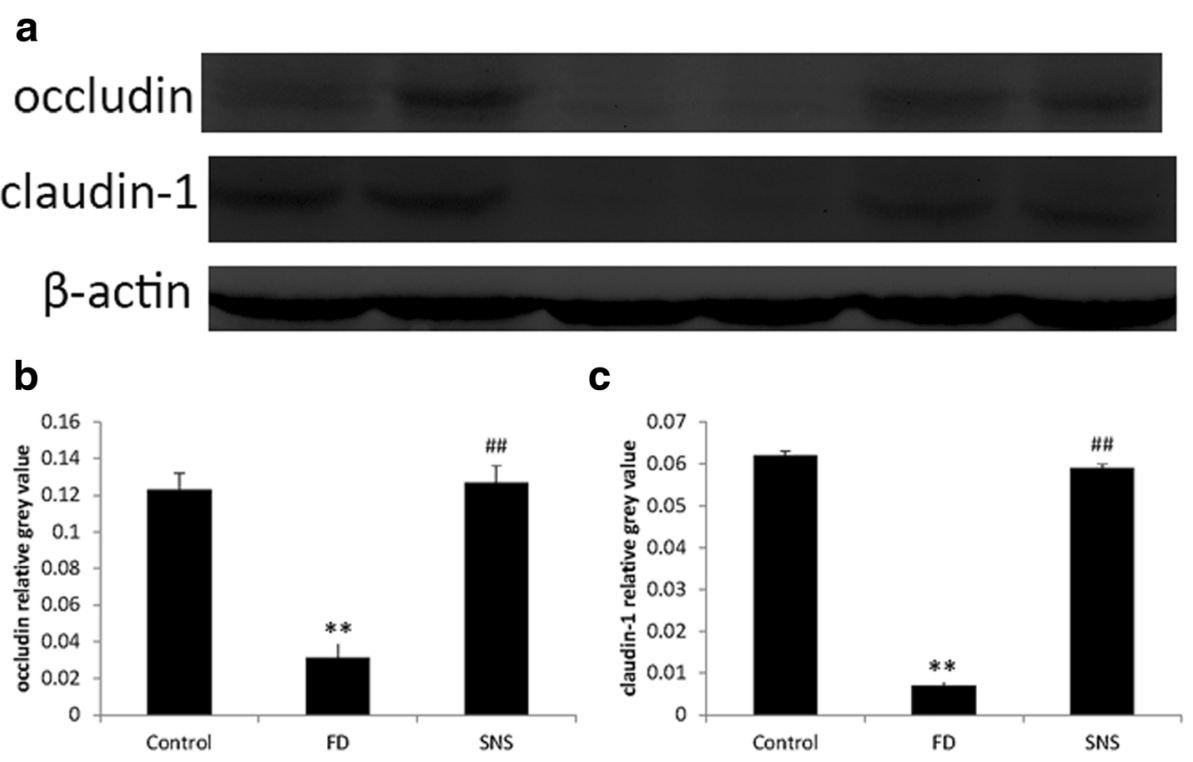

Fig. 3 Representative Western blot bands of TJ proteins (a).The expression level was normalized to the housekeeping protein $\beta$-actin (b, c). Compared with the control group, the expression of occludin and claudin-1 was significant lower in the model group and SNS treatment could restore these two TJ proteins. All data were represented as mean \pm SEM ( 6 rats in each group). ${ }^{* *} P<0.01$ compared with the control group; ${ }^{\# \#} P<0.01$ compared with the model group

TNF- $\alpha$ and IFN- $\gamma$ levels were significantly higher in the duodenum of FD rats than in the control (TNF- $\alpha$ : $1.66 \pm 0.12$ vs. $0.76 \pm 0.05, P<0.001$; IFN- $\gamma: 0.41 \pm 0.01$ vs.0.13 $\pm 0.02, P<0.01)$. Importantly, upon SNS treatment, FD rats showed a much lower level of TNF- $\alpha$ and IFN- $\gamma$ (TNF- $\alpha$ : $0.96 \pm 0.12$ vs. $1.66 \pm 0.12, P<0.01$; IFN- $\gamma: 0.13 \pm 0.01$ vs. $0.41 \pm 0.01, P<0.01$ ), indicating that SNS could ameliorate micro-inflammation in the duodenum of FD rats (Fig. 5).

\section{Discussion}

Our study demonstrated that SNS treatment attenuates FD-associated VH and improves gastric compliance. The underlying therapeutic mechanisms are that SNS improves duodenal barrier integrity by increasing occludin and claudin-1 expression and suppresses microinflammation as suggested by decreased expression of TNF- $\alpha$ and IFN- $\gamma$ in the duodenum.

The intestinal barrier is critical in preventing the translocation of noxious substances and dietary antigens from the gut lumen to the submucosa. Although a large body of evidence has clearly demonstrated intestinal barrier dysfunction is closely related to intestinal diseases including ulcerative colitis [22] and irritable bowel syndrome (IBS) [23], a pathologic relationship between duodenal barrier and FD has only been recognized recently $[2,24]$. Our finding of decreased TEER in FD rats is in accordance with previous reports. The TJ proteins are essential components of the intestinal barrier, and consist of transmembrane proteins, such as occludin, claudin-1, and scaffolding proteins, such as zonula occludens (ZO) [19]. Claudins are the key factors of the paracellular seal, by interacting with ZO-1 or selforganization [25]. Occludin was the first identified T) transmembrane protein [26]. Knockdown of cccludin results in an increase of permeability and the downregulation of occludin was implicated in intestinal diseases [27]. The mechanisms by which tail clamping induces barrier dysfunction remains unclear; however, clinical trials and animal experiments both showed that acute stress has a significant influence on the intestinal inflammatory response and the expression of $\mathrm{TJ}$ proteins $[28,29]$. Of note, we observed that the expression of occludin and claudin-1 was significantly decreased in FD rats. It is likely that the decrease in occludin and claudin-1 expression in the duodenum of FD rats is induced by tail clamping-associated stress through yet unknown mechanisms, such as changes in hormone secretion and/or signaling transduction. The most important finding of the present study is that oral administration of SNS enhances the expression of TJ protein occludin and claudin- 1 in the duodenum, which highlights the potential molecular basis for the therapeutic role of SNS in FD treatment. One of the potential mechanisms is that SNS treatment inhibits proteolysis of occludin by suppressing the transcription of matrix metalloproteinase (MMP) [30, 31].

The impairment of duodenal integrity leads to abnormal penetration of toxic substances, including bacterial metabolites and dietary antigens, which result in activation of 


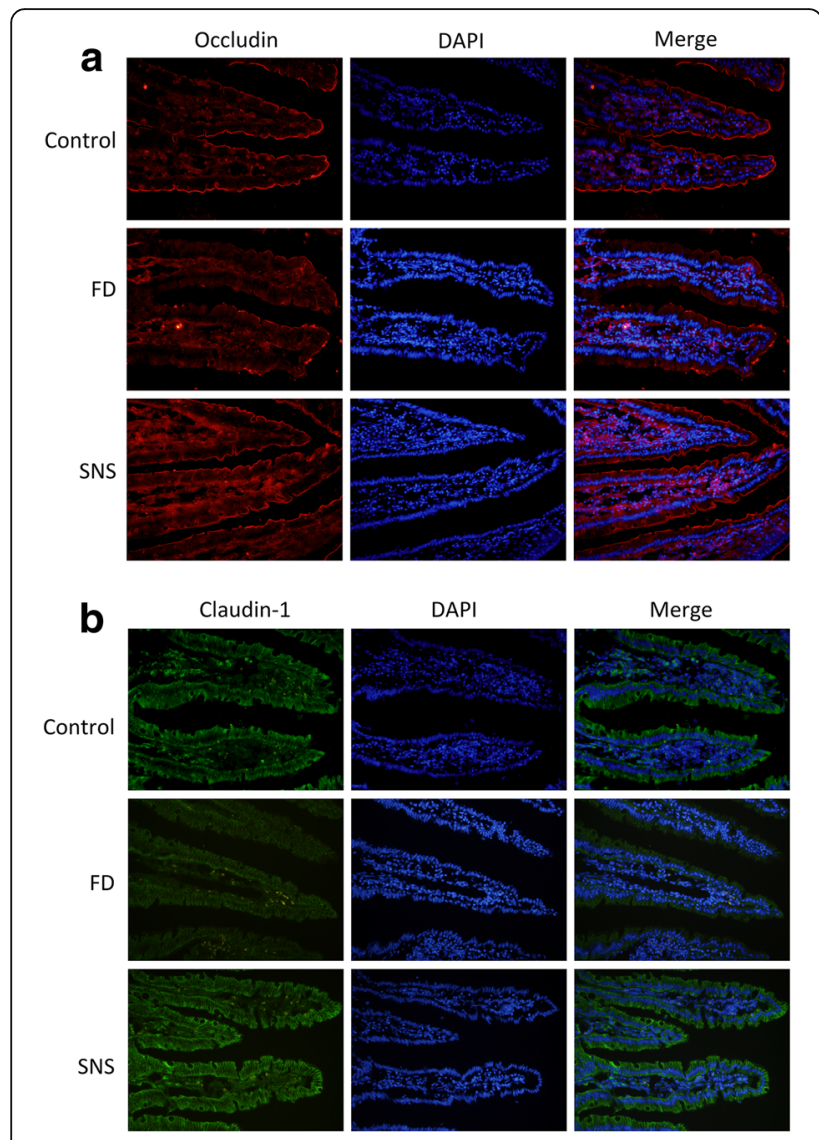

Fig. 4 Representative photomicrographs of immunofluorescence of occluding (a) and claudin-1 (b). These pictures showed a decline in the expression of both occluding and claudin-1 in the model group, which was restored in response to SNS treatment

the autoimmune system and increased infiltration of inflammatory cells. Many studies have demonstrated the abnormal infiltration of mast cells, eosinophil, and intraepithelial lymphocytes in FD patients [2, 21, 29, 32]. Immune cell infiltration-mediated inflammatory response might account for our current observation of increased amount of TNF- $\alpha$ and IFN- $\gamma$. This finding in murine model is consistent with a previous report of human FD patients. Given the stimulatory effect of SNS on the expression of occludin and claudin-1, it is likely that decreased expression of inflammatory cytokines in SNSadministered FD rats results from barrier restoration. However, we can not rule out the possibility that SNS exerts direct effects on immune cell behavior attenuating inflammatory response. It has previously been reported that SNS decreases TNF- $\alpha$ expression in trinitrobenzene sulfonic acid-induced pancreatitis [33], which does not seem to involve barrier restoration. Future study by measuring temporal changes of TEER vs. inflammatory cytokine expression during SNS treatment should provide a better clue. It is also important to test if SNS application in human FD patients enhances duodenal barrier function, and to understand the precise mechanisms of SNS-mediated upregulation of occludin and claudin-1 expression by using animal or cell culture models.

Notably, another key finding of our study is that SNS attenuates FD-associated VH. TRPV1 is a primary causal factor for $\mathrm{VH}$, and many studies have demonstrated the positive co-relationship between TRPV1 expression and VH [34]. It remains unclear how SNS treatment attenuates TRPV1 expression and improves VH in FD rats. It has previously been shown that the duodenum with impaired TJ shows hypersensitivity to acid and lipid $[16,17]$, which can provoke visceral afferents [18]. Moreover, a leaky TJ leads to the invasion of luminal microflora and their metabolites that stimulate immune response as we observed in the current study the increased expression of TNF- $\alpha$ and IFN- $\gamma$. In fact, it has been demonstrated that the expression of TRPV1 is up-regulated in inflammation [35], and TRPV1 can further augment inflammation by activating STAT3 signaling
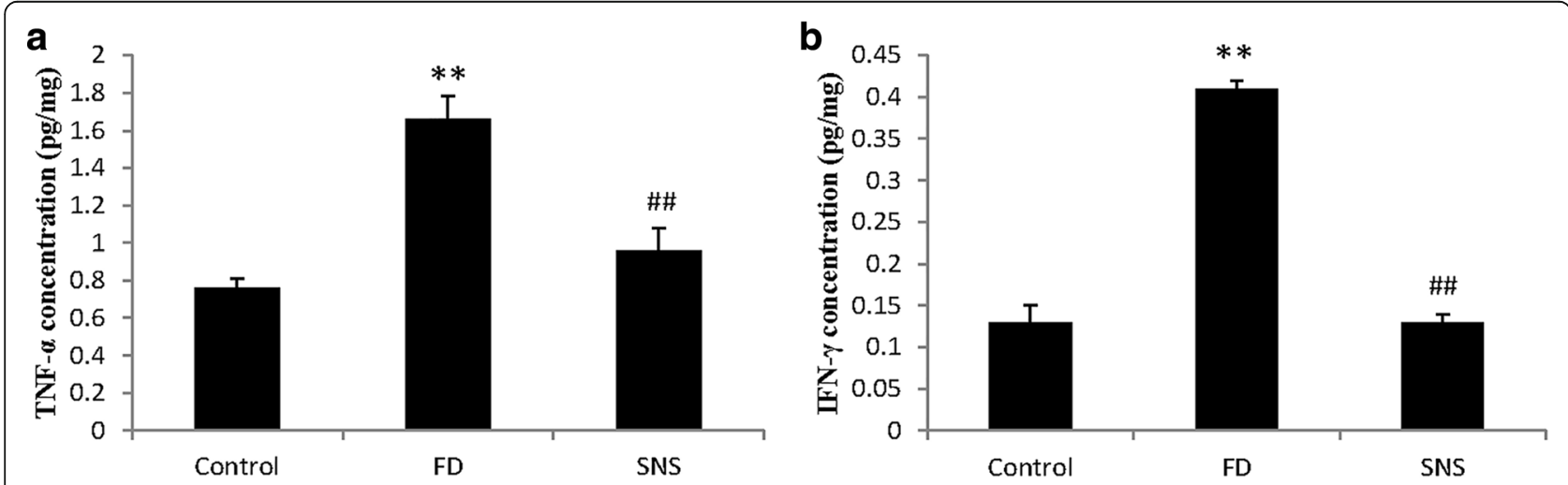

Fig. 5 Concentrations of TNF-a (a) and IFN- $\gamma(\mathbf{b})$ in the duodenum. TNF-a and IFN- $\gamma$ levels were remarkably higher in the duodenal mucosa of model group than the control group. The SNS group showed a lower level of expression of TNF-a and IFN- $\gamma$.. All data were expressed as mean \pm SEM (6 rats in each group). ${ }^{* *} P<0.01$ compared with the control group; ${ }^{\# \# ~} P 0.01$ compared with the model group 
[36]. Therefore, we speculate that restored TJ function in response to SNS has an important role in ameliorating $\mathrm{VH}$ and FD symptoms.

\section{Conclusions}

Our study demonstrated that increased expression of TRPV1, decreased expression of TJ proteins, and the subsequent low-grade inflammation are associated with FD. Importantly, we showed for the first time that Chinese herbal medicine SNS ameliorates the severity of FD possibly through restoration of normal duodenal barrier function by up-regulating occludin and claudin-1 expression and attenuation of local low-grade inflammation as evidenced by decreased secretion of TNF- $\alpha$ and INF- $\gamma$. Our findings thus shed novel light on the molecular basis that underlies SNS-mediated therapeutic effects in FD treatment.

\section{Additional file}

Additional file 1: Dataset. (XLSX $13 \mathrm{~kb})$

\begin{abstract}
Abbreviations
ELISA: Enzyme-linked immunesorbent assay; FD: Functional dyspepsia; IBS: Irritable bowel syndrome; IFN- $\gamma$ : Interferon gamma; MMP: Matrix metalloproteinase; SNS: Sini-San; TCM: Traditional Chinese medicine; TEER: Transepithelial electrical resistance; TJ: Tight junction; TNF-a: Tumor necrosis factor alpha; TRPV1: Transient receptor potential vanilloid type 1
\end{abstract}

\section{Acknowledgments}

We would like to thank Dr. Peijian He at Emory University for his helpful discussion and language editing.

\section{Funding}

This research was supported by the National Natural Science Foundation of China (No.81503514) and Beijing Municipal Administration of Hospitals' Youth Program (No. QML20161002).

\section{Availability of data and materials}

All data generated or analyzed during this study are included in this published article [Additional file 1].

\section{Authors' contributions}

ZSS was responsible for the study concept and design; CXF and ZLQ performed the experiments, collected the data and drafted the manuscript; WJJ performed the experiment and analyzed the data; LXF provided guidance for Ussing chamber use and the barostat experiment, and prepared the graphs. All authors read and approved the final manuscript.

\section{Ethics approval}

This Animal experiment was performed in accordance with the Guide for the Care and Use of Laboratory Animals published by the National Institutes of Health (NIH Publications No. 85-23, revised 1996) and approved by the Animal Care and Use Committee of China Academy of Traditional Chinese Medicine.

\section{Consent for publication}

Not applicable

\section{Competing interests}

The authors declare that they have no competing interests.

\section{Publisher's Note}

Springer Nature remains neutral with regard to jurisdictional claims in published maps and institutional affiliations.

\section{Author details}

${ }^{1}$ Beijing University of Chinese Medicine, Beijing, China. ${ }^{2}$ Digestive Disease Center, Beijing Hospital of Traditional Chinese Medicine Affiliated to Capital Medical University, Beijing, China.

Received: 10 March 2017 Accepted: 18 August 2017

Published online: 30 August 2017

\section{References}

1. Mahadeva S, Goh KL. Epidemiology of functional dyspepsia: a global perspective. World J Gastroentero. 2006;12(17):2661-6.

2. Vanheel H, Vicario M, Vanuytsel T, Van Oudenhove L, Martinez C, Keita AV, et al. Impaired duodenal mucosal integrity and low-grade inflammation in functional dyspepsia. Gut. 2014;63(2):262-71.

3. Stanghellini V, Chan FK, Hasler WL, Malagelada JR, Suzuki H, Tack J, et al. Gastroduodenal Disorders. Gastroenterology. 2016;150(6):1380-92.

4. Miner-Williams WM, Moughan PJ. Intestinal barrier dysfunction: implications for chronic inflammatory conditions of the bowel. Nutr Res Rev. 2016;29(1):40-59.

5. Jin ZH, Duan JH, Zhao HC. Clinical study on colonic transmission time and the effect of sini powder on it in functional constipation patients. Chin J Integr Trad West Med. 2006;26(10):896-8.

6. Chen L. In the treatment of functional dyspepsia with Si-ni-san depression random parallel control study. J Pract Tradit Chin Intern Med. 2014;28(2):50-1.

7. Wang X-M, Wang C-D. Clinical observation of Si-ni-san decoction treated 180 cases patients with functional dyspepsia. Modern Digestion Intervention. 2007;12(4):251-2.

8. FU Kemo MY. Protection effect of Jiaweisini decoction on function of liver and intestinal mucosa of cirrhosis patients. China Moden Doctor. 2014:52(10):105-7.

9. Hu L-N, Fang X-Y, Liu H-L, Gao Z, Wu X-F, Sun Y, et al. Protective effects of 18ß-glycyrrhetinic acid on LPS-induced injury in intestinal epithelial cells. Chin J Nat Med. 2014;11(1):24-9.

10. Wen J, Qiao Y, Yang J, Liu X, Song Y, Liu Z, et al. UPLC-MS/MS determination of paeoniflorin, naringin, naringenin and glycyrrhetinic acid in rat plasma and its application to a pharmacokinetic study after oral administration of Si-Ni-san decoction. J Pharmaceut Bio Med. 2012;66:271-7.

11. Sun $Y$, Dong $Y$, Jiang HJ, Cai $\Pi$, Chen $L$, Zhou $X$, et al. Dissection of the role of paeoniflorin in the traditional Chinese medicinal formula Si-Ni-san against contact dermatitis in mice. Life Sci. 2009;84(11):337-44.

12. Zhang $L$, Dong $Y$, Sun $Y$, Chen $T, X u$ Q. Role of four major components in the effect of Si-Ni-san, a traditional Chinese prescription, against contact sensitivity in mice. J Pharm Pharmacol. 2006:58(9):1257-64.

13. Wei W, Li X, Hao J, Zhang R, Guo J, Zong Y, et al. Proteomic analysis of functional dyspepsia in stressed rats treated with traditional Chinese medicine "Wei Kangning". J Gastroen hepatol. 2011;26(9):1425-33.

14. Uchida M, Kobayashi O, Iwamoto C. Effects of L-tryptophan on gastric emptying evaluated by breath test in relation to gastric accommodation evaluated by Barostat in rats. J Pharmacol Sci. 2015:127(2):229-31.

15. Liu J, Li F, Tang XD, Ma J, Ma X, Ge DY, et al. XiangshaLiujunzi decoction alleviates the symptoms of functional dyspepsia by regulating brain-gut axis and production of neuropeptides. BMC Complem Altern M. 2015;15:387.

16. Bratten J, Jones MP. Prolonged recording of duodenal acid exposure in patients with functional dyspepsia and controls using a radiotelemetry $\mathrm{pH}$ monitoring system. J Clin Gastroenterol. 2009;43(6):527-33.

17. Feinle C, Meier O, Otto B, D'Amato M, Fried M. Role of duodenal lipid and cholecystokinin a receptors in the pathophysiology of functional dyspepsia. Gut. 2001:48(3):347-55

18. Schwartz MP, Samsom M, Smout AJ. Chemospecific alterations in duodenal perception and motor response in functional dyspepsia. Am J Gastroentero. 2001;96(9):2596-602.

19. Van Itallie CM, Anderson JM. Architecture of tight junctions and principles of molecular composition. Semin Cell Dev Biol. 2014;36:157-65.

20. Walker MM, Talley NJ. The role of duodenal inflammation in functional dyspepsia. J Clin Gastroenterol. 2017;51(1):12-8. 
21. Du L, Shen J, Kim JJ, Yu Y, Ma L, Dai N. Increased duodenal eosinophil degranulation in patients with functional dyspepsia: a prospective study. Sci Rep-UK. 2016;6:34305.

22. Schmitz H, Barmeyer C, Fromm M, Runkel N, Foss HD, Bentzel CJ, et al. Altered tight junction structure contributes to the impaired epithelial barrier function in ulcerative colitis. Gastroenterology. 1999;116(2):301-9.

23. Wilcz-Villega EM, McClean S, O'Sullivan MA. Mast cell tryptase reduces junctional adhesion molecule-a (JAM-A) expression in intestinal epithelial cells: implications for the mechanisms of barrier dysfunction in irritable bowel syndrome. Am J Gastroenterol. 2013;108(7):1140-51.

24. Tanaka F, Tominaga K, Fujikawa Y, Nagami Y, Kamata N, Yamagami H, et al. Concentration of glial cell line-derived neurotrophic factor positively correlates with symptoms in functional dyspepsia. Digest Dis Sci. 2016;61(12):3478-85.

25. Gunzel D, Yu AS. Claudins and the modulation of tight junction permeability. Physiol Rev. 2013;93(2):525-69.

26. Furuse $\mathrm{M}$, Hirase $\mathrm{T}$, Itoh $\mathrm{M}$, Nagafuchi $\mathrm{A}$, Yonemura S, Tsukita $\mathrm{S}$, et al. Occludin: a novel integral membrane protein localizing at tight junctions. J Cell Biol. 1993;123:1777-88.

27. Feldman GJ, Mullin JM, Ryan MP. Occludin: structure, function and regulation. Adv Drug Deliver Rev. 2005;57(6):883-917.

28. Lee HS, Kim DK, Kim YB, Lee KJ. Effect of acute stress on immune cell counts and the expression of tight junction proteins in the duodenal mucosa of rats. Gut live. 2013;7(2):190-6.

29. Vanuytsel T, van Wanrooy S, Vanheel H, Vanormelingen C, Verschueren $S$, Houben E, et al. Psychological stress and corticotropin-releasing hormone increase intestinal permeability in humans by a mast cell-dependent mechanism. Gut. 2014;63(8):1293-9.

30. Lin HJ, Kao ST, Siao YM, Yeh CC. The Chinese medicine Sini-san inhibits $\mathrm{HBX}$-induced migration and invasiveness of human hepatocellular carcinoma cells. BMC Complem Altern M. 2015;15:348.

31. Yang F, Zhao K, Zhang $X$, Zhang J, Xu B. ATP induces disruption of tight junction proteins via IL-1 Beta-dependent MMP-9 activation of human blood-brain barrier in vitro. Neural Plast. 2016;8928530

32. Talley NJ, Walker MM, Aro P, Ronkainen J, Storskrubb T, Hindley LA, et al, Non-ulcer dyspepsia and duodenal eosinophilia: an adult endoscopic population-based case-control study. Clin Gastroenterol H. 2007;5(10):1175-83.

33. Tang Y, Liao Y, Kawaguchi-Sakita N, Raut V, Fakhrejahani E, Qian N, et al. Sinisan, a traditional Chinese medicine, attenuates experimental chronic pancreatitis induced by trinitrobenzene sulfonic acid in rats. J Hepato-BilPan Sci. 2011:18(4):551-8

34. Farzaei MH, Bahramsoltani R, Abdollahi M, Rahimi R. The role of visceral hypersensitivity in irritable bowel syndrome: pharmacological targets and novel treatments. J Neurogastroenterol Motil. 2016;22(4):558-74.

35. Ma W, Quirion R. Inflammatory mediators modulating the transient receptor potential vanilloid 1 receptor: therapeutic targets to treat inflammatory and neuropathic pain. Expert Opin Ther Tar. 2007:11(3):307-20.

36. Yoshida A, Furube E, Mannari T, Takayama Y, Kittaka H, Tominaga M, et al. TRPV1 is crucial for proinflammatory STAT3 signaling and thermoregulationassociated pathways in the brain during inflammation. SCI REP-UK. 2016;6:26088.

\section{Submit your next manuscript to BioMed Central and we will help you at every step:}

- We accept pre-submission inquiries

- Our selector tool helps you to find the most relevant journal

- We provide round the clock customer support

- Convenient online submission

- Thorough peer review

- Inclusion in PubMed and all major indexing services

- Maximum visibility for your research

Submit your manuscript at www.biomedcentral.com/submit

) Biomed Central 